\title{
Effect of Temperature on the Circular Dichroism Spectra of Polypeptides in the Extended State
}

\author{
M. LOIS TIFFANY and S. KRIMM, Biophysics Research Division, \\ Institute of Science and Technology, and Harrison M. Randall Laboratory \\ of Physics, University of Michigan, Ann Arbor, Michigan 48104
}

\begin{abstract}
Synopsis
The circular dichroism (CD) spectra of poly-L-proline and of poly-trglutamic acid and poly-L-lysine in their charged states have been studied as a function of temperature. The variation of CD spectra with temperature is inconsistent with the assignment of the speetrum of such charged polypeptides to an unordered chain conformation, but does support our earlier assignment to a locally ordered structure-what we have called the extended helix conformation. These results also strengthen our previous assignment of the CD spectrum of an unordered chain, and indicate that three conformational states ( $\alpha$-helix, extended helix, and unordered) should be incorporated in our thinking about conformational transitions in polypeptides.
\end{abstract}

\section{INTRODUCTION}

The circular dichroism (CD) spectra of regular polypeptide chain structures, such as the $\alpha$-helix, $\beta$ pleated sheet, polyprolines I and II, and collagen, are satisfactorily characterized and well accepted. There has been controversy, however, over the assignment of the CD spectrum of the unordered polypeptide chain.

Because the $\mathrm{pH}$-induced cooperative transitions in many properties of poly-L-glutamic acid (PGA) and poly-L-lysine (PL) were taken to correspond to a helix-to-coil transformation, the CD spectra of the charged forms of these polypeptides was initially believed ${ }^{1}$ to represent that of a random coil. It was subsequently pointed out ${ }^{2}$ that the spectrum of denatured collagen did not correspond to that of the charged polypeptides: while the latter had an intense negative band near $200 \mathrm{~nm}$ and a weak positive band near $220 \mathrm{~nm},{ }^{3}$ denatured collagen was characterized by a much weaker negative band near $200 \mathrm{~nm}$ and no positive band at longer wavelengths. Denatured collagen was considered to be a superior model system for an unordered chain, ${ }^{2}$ and this view received support when it became possible to produce similar spectra with poly-L-proline (PP) in concentrated aqueous $\mathrm{CaCl}_{2}$ solution. ${ }^{4}$ That this is a spectrum associated with a new chain conformation and not with a specific salt interaction effect was shown by the fact that the same spectrum resulted from a variety of treatments. ${ }^{5}$ This has been more recently confirmed by the fact that $6 \mathrm{M} \mathrm{CaCl} \mathrm{Cl}_{2}$ has no 
effect on the CD spectrum of a rigid model compound. ${ }^{6}$ Finally, both PGA and PL could also be shown to give such spectra in the presence of salts. ${ }^{5}$

If, then, the CD spectra of charged PGA and PL are not to be attributed to unordered polypeptide chains, what is their structural origin? We had suggested ${ }^{3}$ that this spectrum probably arises from a local, relatively ordered backbone conformation, one which in this case would be expected to result from the repulsive interactions between side chain charges. This conformation, a roughly threefold lefthanded helical structure ${ }^{3}$ was subsequently shown to be a reasonable one from energy calculations. ${ }^{7}$ It also was consistent with the effect of counterions and non-aqueous solvents on the spectrum. ${ }^{8}$ It should be noted that we did not claim that electrostatic repulsions were the only interactions that would give rise to this extended helical (EH) structure. ${ }^{5}$ Evidence has since been obtained ${ }^{9}$ that a wide variety of factors, such as low temperature, side-chain steric hindrance in the absence of intrachain hydrogen bonding, protonation of the backbone by strong acids, interaction of urea and guanidine hydrochloride with the backbone carbonyls, and interaction of monovalent cations with the carbonyls, can also lead toward the EH state. The fact that the CD spectrum of poly [ $N^{5}$-(2-hydroxyethyl)-L-glutamine] in water ${ }^{10}$ resembles that of charged PGA is therefore not an inconsistency, as is assumed by the above authors. ${ }^{11}$ Our assignment has received additional strong experimental support from the observation ${ }^{12}$ that poly(ala gly gly) can be dem-

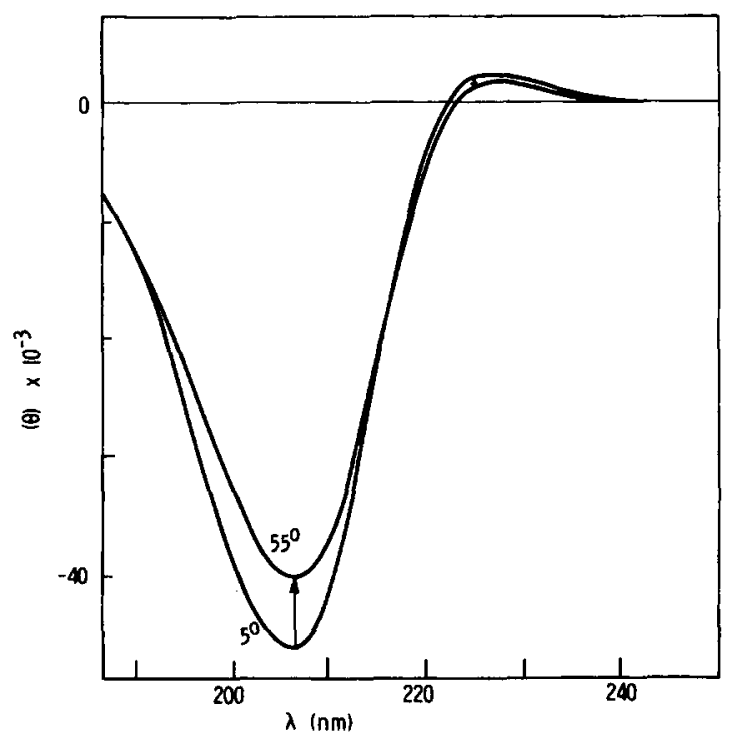

Fig. 1. Circular dichroism spectrum of poly-L-proline II (Sigma, molecular weight $55,000)$ as a function of temperature. This and all subsequent spectra were recorded in temperature jacketed cells, using a Sproul modified Durrum-Jasco ORD-UV-5 recording spectrometer. 
onstrated to have an $\mathrm{EH}$ structure and to give a $\mathrm{CD}$ spectrum similar to that of charged PGA and PL.

Despite the above evidence, it has still been argued from theoretical ${ }^{13,14}$ and experimental ${ }^{11,15-18}$ studies that the CD spectrum of charged PGA or PL corresponds to that of a random chain. The force of the theoretical arguments is removed by more detailed calculations, ${ }^{19,20}$ which show that the predicted spectrum of the unordered chain in fact does not agree with that observed for the charged models, but is completely consistent with that proposed by us.,5,9 The experimental studies introduce no new relevant arguments, and therefore derive their assignment from the earliest proposal. ${ }^{1}$

It is clear that any additional experimental evidence and arguments relating to the assignment of the CD spectrum of charged PGA and PL will be of value in helping to resolve the above problem. In this connection we have studied the effect of temperature on the CD spectra of some polypeptides which we have suggested are in the EH structure. We belicve that the results of these studies support this assignment and confirm that the suggested alternate assignment to an unordered chain is incorrect. In addition, they throw light on the role of the unordered chain in conformational transitions in polypeptides.

\section{RESULTS AND DISCUSSION}

1. Poly-L-proline. The $3_{1}$ lefthanded helical structure of PP II can serve as a model for the EH conformation in polypeptides, particularly if account is taken of the peak shifts resulting from the difference in chromophore $^{3,5,12}$ We have therefore examined the CD spectrum of PP II as a function of temperature, and the results are shown in Figure 1 for the range of $5^{\circ}$ to $55^{\circ} \mathrm{C}$. The change is not very large, but it is seen that the bands are definitely more intense at the lower temperature. The question arises as to the structural basis of this spectral effect.

Many arguments suggest that the above spectral intensification arises from a greater chain rigidity at the lower temperature which gives rise to an effectively longer spectroscopic unit. Experimental studies on model compounds show that the CD spectra of rigid molecules are, in general, more intense than those of flexible molecules. ${ }^{21}$ Theoretical studies of CD spectra as a function of helix length ${ }^{19,22}$ also confirm that the dichroism per residue is greater the longer the helix. In the case of PP it has been calculated that the binding of water to the imide group tends to rigidify the chain. ${ }^{23}$ The heating of a solution of PP II leads to a weakening interaction between the chain and the bound water, the PP II in fact, ultimately precipitating as a crystalline solid at high enough temperatures. ${ }^{24,25}$ At the same time, elevated temperatures also contribute to enhanced chain motion and flexibility. Thus, all of the evidence points to a "loosening" of the $3_{1}$ structure PP II as the temperature is raised, leading to a weakening of the bands in the CD spectrum. 


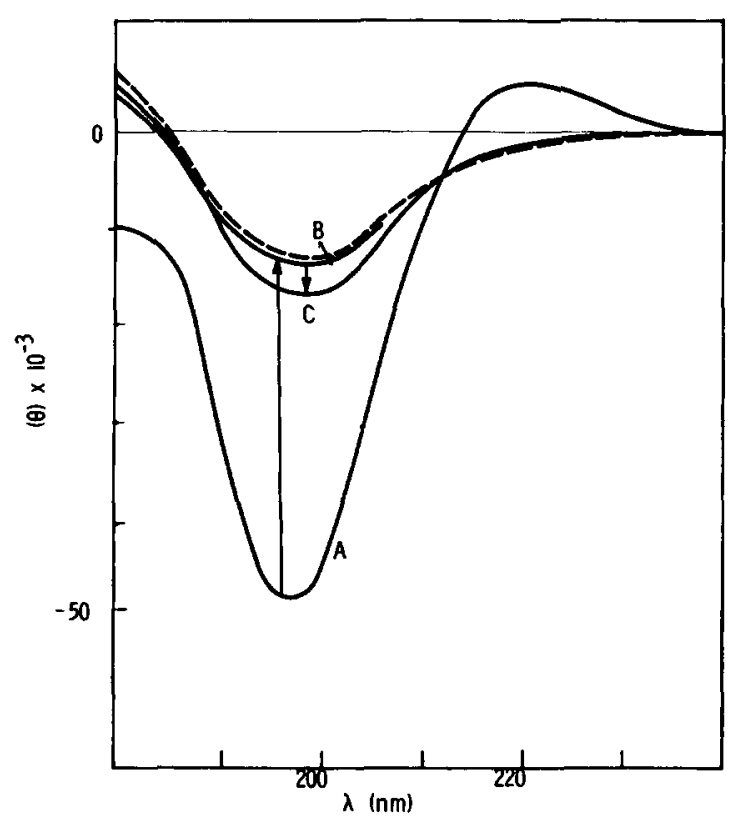

Fig. 2. Circular dichroism spectrum of collagen (rat tail tendon) as a function of temperature. (A) native; (B) heat-denatured, at $60^{\circ} \mathrm{C} ;(\mathrm{C})$ same sample as (B), cooled to $25^{\circ} \mathrm{C}$ and run immediately. (- - is the spectrum of a film prepared from (B).)

It should be noted that this intensity change cannot be attributed to changes in solution volume with temperature: for the $50^{\circ} \mathrm{C}$ change shown in Figure 1 the intensity change is expected ${ }^{26}$ to be about $7 \%$, whereas the actual change is about $15 \%$. This is further substantiated by studies on collagen (compare Fig. 2). We find (see Fig. 2A) that when native collagen is heated from $5^{\circ}$ to $\sim 37^{\circ} \mathrm{C}$ no change occurs in the CD spectrum (the volume change over this temperature interval is expected to be about $4 \%$, which is within experimental error). Furthermore, any general changes in bandwidth with temperature must be minimal over such a temperature range, since we see no significant change in the CD spectrum. It appears that the integrity of the triple-helical structure is maintained prior to denaturation, and in the absence of conformational changes the CD spectrum is influenced relatively little by temperature. If collagen is heated above $40^{\circ} \mathrm{C}$ it denatures, and the $\mathrm{CD}$ spectrum is a good approximation to an unordered chain ${ }^{2,5}$ (compare Fig. 2B). Similar results have been obtained recently. ${ }^{27}$ The $\mathrm{CD}$ spectrum is somewhat sensitive to temperature (compare Fig. $2 \mathrm{C}$ ), in a manner consistent with early optical rotation measurements on many molecules, ${ }^{28}$ but this probably arises from small changes in conformational equilibrium with temperature. As we have noted before, ${ }^{5}$ there is no single characteristic unordered structure, and therefore CD spectra are expected to vary somewhat, particularly in band intensities, as a function of the details of chain conformation. It is interesting to note that the CD spectrum of collagen is positive below about $184 \mathrm{~nm}$. This 


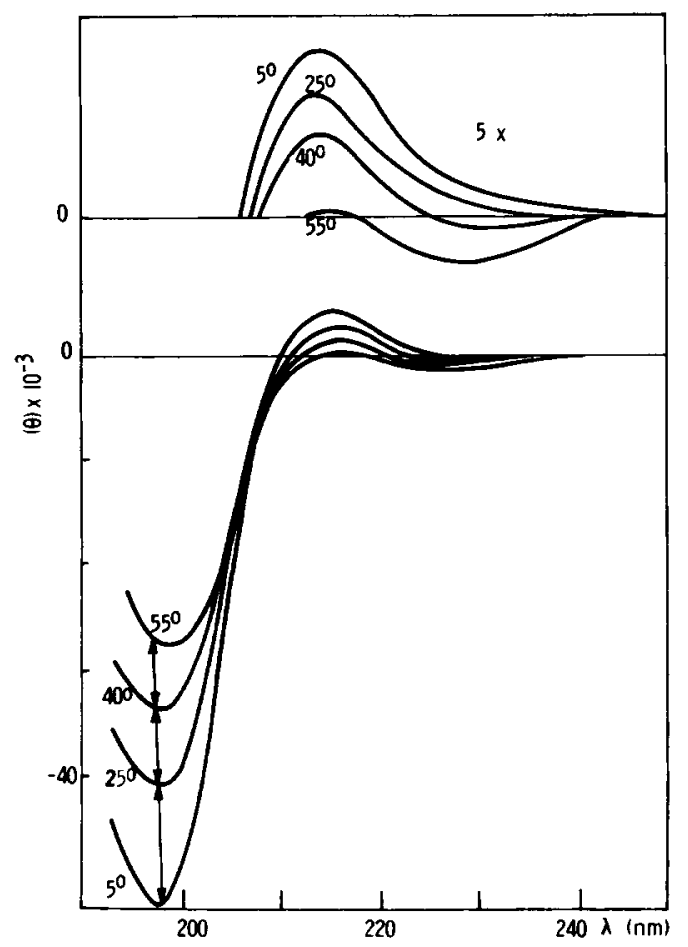

Fig. 3. Circular dichroism spectrum of poly-L-glutamic acid, New England Nuclear (at pH 7) as a function of temperature.

had not been reported before, but is consistent with the form of CD curve predicted $^{19,20}$ for an unordered chain.

2. Poly-L-glutamic Acid. The effect of temperature on the CD spectrum of charged PGA is shown in Figure 3. Again we see that lowering the temperature results in an intensification of the spectrum, a result which, by analogy with PP II, we interpret as arising from a rigidifying of the structure. At elevated temperatures the spectrum weakens, as well as changing its shape.

These results reveal the difficulty in attributing the characteristic spectrum of charged PGA to an uniordered chain conformation. If such an assignment were appropriate to the $5^{\circ} \mathrm{C}$ spectrum, then we face the paradoxical situation that raising the temperature to $25^{\circ} \mathrm{C}$, which should give rise to a more random structure results in a weakening of the spectrum. In fact, as the temperature is raised to $55^{\circ} \mathrm{C}$ (see Fig. 3) the spectrum departs dramatically from its form at $5^{\circ} \mathrm{C}$. This is hardly consistent with an assignment of the $5^{\circ}$ spectrum to an unordered chain, but this behavior is in agreement with an assignment to a more regular, viz., the $\mathrm{EH}$, structure.

The above temperature behavior, on the other hand, is entirely consistent with our previous assignment of the CD spectrum of the unordered polypeptide chain. ${ }^{4,5}$ As the temperature is raised, we expect that an increasing 


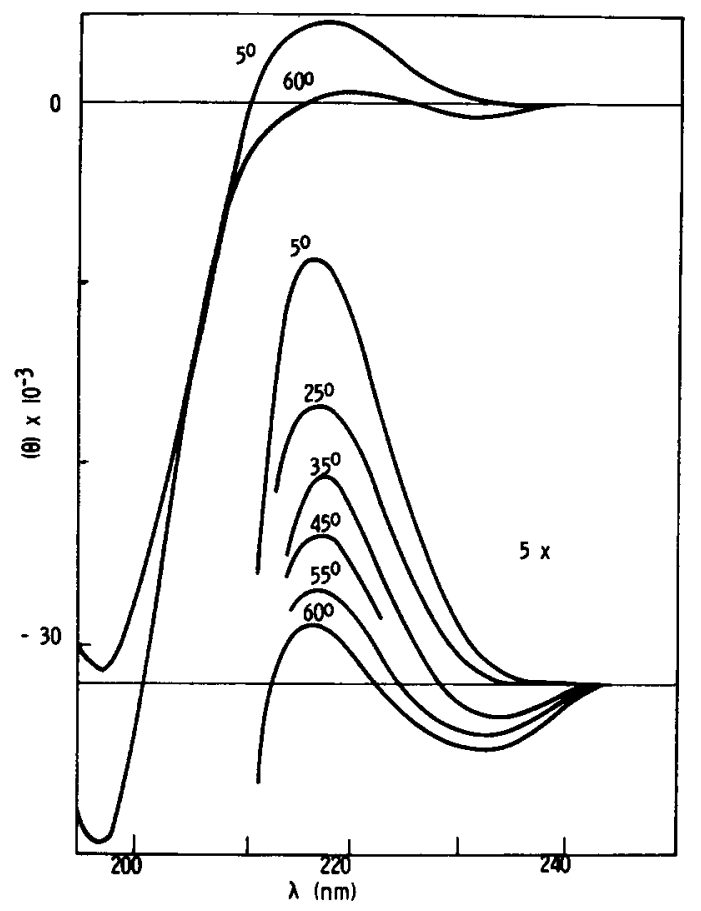

Fig. 4. Circular dichroism spectrum of poly-L-lysine, New England Nuclear (at pH 7 as a function of temperature.

proportion of the chains will develop unordered conformations. The CD spectrum will then consist primarily of a superposition of the CD spectra of unordered and of EH conformations. The exact form of this curve will depend on the detailed CD curves taken for these structures (and, of course, on the possible presence of small amounts of $\alpha$-helix or other specific conformations), but the curves in Figure 3 for $40^{\circ} \mathrm{C}$ and $55^{\circ} \mathrm{C}$ are of the shape and magnitude expected from such a combination.

It should be noted that the above curves are not likely to be a result of a mixture of only $\alpha$-helix and EH conformations. In the latter case, such as conversions produced by a change in $\mathrm{pH}$, there is an isodichroic point at about $204 \mathrm{~nm}$. For the temperature effect there is no isodichroic point but a region of crossover, and this occurs at longer wavelength (206-209 nm). Thus, at least three states are probably involved: the $\alpha$-helix, the $\mathbf{E H}$, and unordered conformations.

3. Poly-L-lysine. The temperature effects on the CD spectrum of the charged form of PL are similar to those for PGA (see Fig. 4). In addition, data on the degree of dissociation of charged PL as a function of temperature ${ }^{29}$ show that this does not change over the temperature range in which we observe changes in the CD spectrum. This shows that the latter changes do not arise from a change in $\mathrm{pK}$ with temperature (which we also assume to be the case for PGA). It should be noted that although no changes in optical rotatory dispersion with temperature were observed, ${ }^{29}$ 


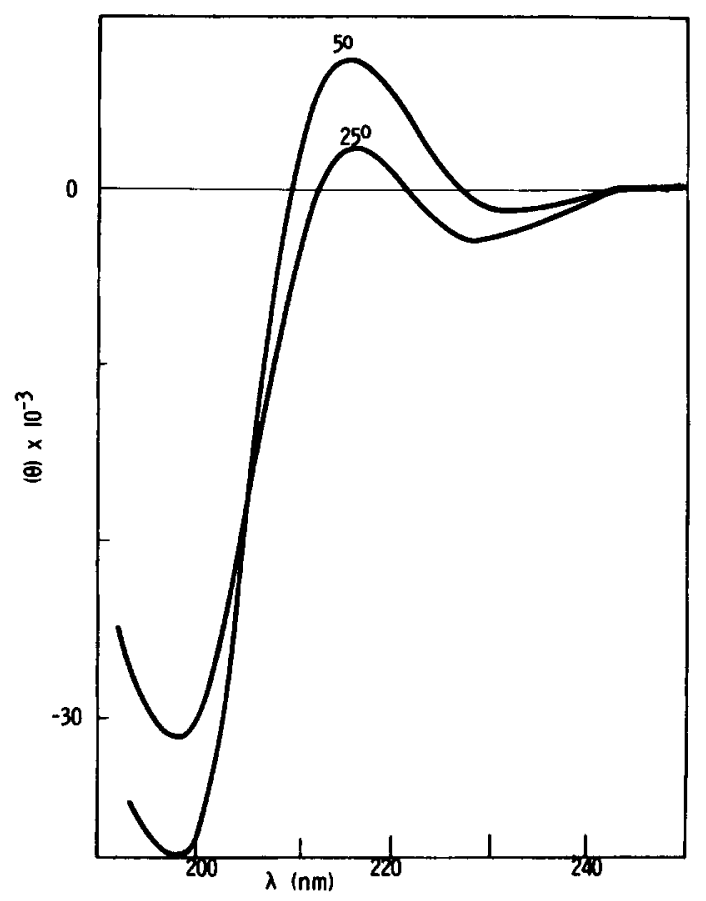

Fig. 5. Circular dichroism spectrum of poly-L-aspartic acid, New England Nuclear (at $\mathrm{pH} 7)$ as a function of temperature. $\left(5^{\circ} \mathrm{C}\right.$ curve is below $55^{\circ} \mathrm{C}$ at $\lambda=198 \mathrm{~nm}$.)

this is not inconsistent with our results since $C D$ is a much more sensitive technique.

4. Poly-L-aspartic Acid. This polypeptide, when charged, does not give the normal two-band spectrum of the EH conformation ${ }^{3}$ (see Fig. 5). Yet when the temperature is lowered from room temperature to $5^{\circ} \mathrm{C}$ the spectrum of the EH conformation is approached (compare Fig. 5). This behavior is consistent with the interpretation which we have given above.

In conclusion, the temperature variation of the CD spectra of charged PGA and PL is inconsistent with their being in an unordered state but does support their assignment to a local EH conformation. ${ }^{3,5}$ On the other hand, these spectral variations do lend additional support to our earlier assignment of the CD spectrum of the unordered polypeptide chain. ${ }^{4,5}$ It should be noted that the temperature variations of the CD spectra of various sequential polypeptides ${ }^{12,27,30,31}$ are in complete agreement with the conclusions reached here.

The recognition that the unordered and $\mathrm{EH}$ structures are different conformational states has important implications with respect to "helixcoil transitions." It suggests that we should incorporate a third structural state in the considerations of conformational transitions. Thus, the $\mathrm{pH}$-induced transition in PGA or PL involves a transformation between $\alpha$-helical and EH structure, but a certain fraction of the conformations consist of unordered states, the fraction increasing with temperature. Other experimental results, such as the optical behavior of poly- $\gamma$-benzyl-L- 
glutamate in different dichloroacetic acid-ethylene dichloride mixtures as a function of temperature, ${ }^{32}$ also seem best explained by assuming that a truly unordered state is approached only at high temperatures. An extrapolation of the $b_{0}$ data of different homologs of poly [ $N^{5}$-(2-hydroxyalkyl)-Lglutamine] of differing alkyl side chain length as a function of temperature $^{33}$ again indicates the existence of three structural states with the unordered only existing at high temperatures. While a two-state helix-coil theory is clearly more attractive, experimental evidence requires the inclusion of three conformational states in real polypeptide systems.

This research was supported by National Science Foundation grant GB-15682. One of us (M. L. T.) is indebted for support from PHS Training Grant GM-1355 from the National Institute of General Medical Science, NIH.

\section{References}

1. G. Holzwarth and P. Doty, J. Amer. Chem. Soc., 87, 218 (1965).

2. M. Lois Tiffany and S. Krimm, Biophys. Soc. Abstr., TE5 (1967).

3. M. Lois Tiffany and S. Krimm, Biopolymers, 6, 1379 (1968).

4. M. Lois Tiffany and S. Krimm, Biopolymers, 6, 1767 (1968).

5. M. Lois Tiffany and S. Krimm, Biopolymers, 8, 347 (1969).

6. F. Naider, E. Benedetti, and M. Goodman, Proc. Natl. Acad. Sci., 68, 1195 (1971).

7. S. Krimm and J. E. Mark, Proc. Natl. Acad. Sci., 60, 1122 (1968).

8. S. Krimm, J. E. Mark, and M. Lois Tiffany, Biopolymers, 8, 695 (1969).

9. M. Lois Tiffany, Ph.D. Thesis, University of Michigan, 1971.

10. A. J. Adler, R. Hoving, J. Potter, M. Wells, and G. D. Fasman, J. Amer. Chem. Soc., 90, 4736 (1968).

11. G. D. Fasman, H. Hoving, and S. N. Timasheff, Biochemistry, 9, 3316 (1970).

12. W. B. Ripon and A. G. Walton, Biopolymers, 10, 1207 (1971).

13. A. E. Tonelli, Macromolecules, 2, 635 (1969).

14. D. Aebersold and E. S. Pysh, J. Chem. Phys., 53, 2156 (1970).

15. Y. P. Myer, Macromolecules, 2,624 (1969).

16. D. G. Dearborn and D. B. Wetlaufer, Biochem. Biophys. Res. Comm., 39, 314 (1970).

17. D. Balasubramanian and R. S. Roche, Chem. Commun., 862 (1970).

18. J. R. Parrish and E. R. Blout, Biopolymers, 10, 1491 (1971).

19. E. W. Ronish, Ph.D. Thesis, University of Michigan, 1970.

20. E. W. Ronish and S. Krimm, Biopolymers, in press.

21. M. Goodman, C. Toniolo, and J. Falcetta, J. Amer. Chem. Soc., 91, 1816 (1969).

22. R. W. Woody and I. Tinoco, J. Chem. Phys., 46, 4929 (1967).

23. S. Krimm and C. M. Venkatachalam, Proc. Natl. Acad. Sci., 68, 2468 (1971).

24. A. Ciferri and T. A. Orofino, J. Phys. Chem., 70, 3277 (1966).

25. L. Mandelkern and M. H. Liberman, J. Phys. Chem., 71, 1163 (1967).

26. F. R. Brown, J. P. Carver, and E. R. Blout, J. Mol. Biol., 39, 307 (1969).

27. F. R. Brown, A. J. Hopfinger, and E. R. Blout, J. Mol. Biol., 63, 101 (1972).

28. W. Kauzmann, J. E. Walter, and H. Eyring, Chem. Rev., 26, 339 (1940).

29. P. Y. Chou and H. A. Scheraga, Biopolymers, 10, 657 (1971).

30. B. B. Doyle, W. Traub, G. P. Lorenzi, F. R. Brown, and E. R. Blout, J. Mol. Biol., $51,47(1970)$.

31. W. L. Mattice and L. Mandelkern, J. Am. Chem. Soc., 92, 5285 (1970).

32. A. Teramoto, K. Nakagawa, and H. Fujita, J. Chem. Phys., 46, 4197 (1967).

33. N. Lotan, A. Yaron, and A. Berger, Biopolymers, 4, 365 (1966).

Received March 17, 1972

Revised July 5, 1972 